\title{
Facilitating Business to Government Interaction Using a Citizen-Centric Web 2.0 Model
}

\author{
Alexandros Dais, Mara Nikolaidou, and Dimosthenis Anagnostopoulos \\ Harokopio University of Athens, Department of Informatics and Telematics, \\ El. Venizelou 70, 17671, Athens \\ \{adais, mara, dimosthe\} @hua.gr
}

\begin{abstract}
Modelling Business to Government (B2G) interaction is considered to be more complex than Citizen to Government $(\mathrm{C} 2 \mathrm{G})$ interaction, since the concept of authorized citizens, representing the Business while interacting with specific governmental organizations should be explored. Novel interaction models should be introduced, transforming the way Governmental services are delivered to Businesses. To this end, we propose a Web 2.0 citizen-centric model facilitating Business to Government interaction by establishing a social network between citizens and public agencies. All kinds of interactions (B2G, $\mathrm{G} 2 \mathrm{G}$ ) are expressed as $\mathrm{C} 2 \mathrm{G}$ interactions establishing the citizen-centric nature of the proposed interaction model. The architecture of a Web 2.0 platform, named MyCCP, based on the suggested interaction model is also presented, along with a case study illustrating business-to-government interaction to indicate the potential of the suggested model.
\end{abstract}

Keywords: Business to Government interaction model, Citizen Centric Model, Web 2.0, T- Gov.

\section{Introduction}

Whole-of-government approach is the current trend in providing complex crossorganizational e-services to citizens by supporting a "central portal" acting as a "single access point" for all services, either cross-organizational or not [1]. The integration and coordination of existing services, provided by independent public agencies, in an automated and transparent fashion is explored in the case of "active life event portal" [2]. Life events constitute a grouping mechanism of public e-services according to citizen needs. In particular, each life event corresponds to a workflow pre-composed by existing e-services and executed by the citizen as a single application. Such services are currently provided through a "central portal" acting as a "single access point" for all governmental services either cross-organizational or not.

Modelling Business to Government (B2G) interaction is considered to be more complex than Citizen to Government $(\mathrm{C} 2 \mathrm{G})$ interaction, since the concept of an authorized citizen, that represent the Business while interacting with a specific governmental organization should be introduced. Similar to the real world, in the digital world, a business should be considered as a legal entity represented by authorized 
citizens when interaction with the state. Thus, new requirements are imposed when modelling B2G interaction, not effectively handled in case of complex crossorganizational services, where the Business is represented by different citizens when interacting with different governmental agencies. In such case, relations between businesses, citizens, as well as among government agencies, should be established and managed. Furthermore more effective interaction models are needed for cross organizational integration, while innovative service delivery and enforcement of privacy and confidentiality should also be considered.

The adoption of the Web 2.0 paradigm to effectively model $\mathrm{B} 2 \mathrm{G}$ interaction is a challenge resulting in the transformation of B2G service delivery. B2G service delivery transformation can be regarded as part of the Transformational Government (T-Gov) concept [3]. T-Gov requirements, as expressed in terms of citizen-centric delivery of public services and effective cross-organizational interoperability, could be fulfilled utilizing Web 2.0 concepts. Though Web 2.0 can not be perceived as a single new technology or standard, it can be outlined by its key features which revolve around the notions of a user built, user-centred and user-run Internet [4]. The most prominent example of the Web 2.0 paradigm is social computing. A new generation of web-based communities are rapidly becoming popular among people worldwide. In fact, an entire generation of young people has come of age using Internet as the dominant medium for socializing. These virtual communities are familiar with the interaction mechanisms provided by the social networking platforms. Eventually, Web 2.0 environment, emphasizing on collaboration and communication, will gradually affect citizens' perception of electronic government and will contribute to the transformation of government structures and services. A corresponding interaction model should take into consideration every kind of relationship between the constituents of T-Gov, namely citizens, business and government agencies, and allow them to communicate in a seamless, simple fashion, similar to one they are used to in real life.

In the following, we introduce a citizen-centric model for $\mathrm{B} 2 \mathrm{G}$ interaction by adopting the Web 2.0 perspective. Basic characteristics of B2G service delivery are defined in section 2. The proposed interaction model is described in section 3. The architecture of a Web 2.0 platform, named MyCCP, based on the suggested interaction model is briefly presented in section 4 . In section 5 a case study is described, illustrating business-to-government interaction to indicate the potential of the suggested model, while conclusions reside in section 6 .

\section{Transforming B2G Service Delivery}

Business to Government (B2G) interaction is considered to be more complex to handle than Citizen to Government (C2G), as businesses are often represented by authorized employees that actually interact with the government to complete a specific task. Those employees are actually intermediates between the Business and the Government. Elaborating the model, we argue that authorized employees are actual citizens performing authorized tasks inside the context of a specific B2G service. So any B2G service should be decomposed to corresponding C2G services forming it. Such decomposition poses new requirements. 
First of all citizens should be authorized to act as intermediates for a business. The authorization process should be formal and legally correct. Non repudiation methods should be enforced to bind citizens to their actions. Furthermore, the authorization mechanism should be agile. Relations between the citizens the business should be handled in a proper way.

Additionally, intermediates should only view the information that is required to accomplish their task and certainly not every kind of business information. Confidentiality and privacy issues should be addressed to prevent unauthorized access to business information. So, data handling is considered a major requirement to be fulfilled.

The distributed nature of $\mathrm{B} 2 \mathrm{G}$ service decomposition requires some sort of central control. In the real word, there is always someone that is liable to governmental laws, acts and regulations as far as the business is concerned.

Last but not least, cross organizational tasks often requires the involvement of many intermediates. So there is a need for the intermediates to collaborate in an asynchronous and distributed way to accomplish a common goal. So, a reliable interaction mechanism between the intermediates should exist.

Furthermore, issues related to Authentication, Data Integrity and Availability should be addressed. While confidentiality deals with the unintentional disclosure of information outside the authorized parameters, data integrity assures the trustworthiness of the information and availability ensures that the information is made available to authorized intermediates.

Taking into account the requirements mentioned before, we propose a CitizenCentric Model for Business to Government interaction utilizing most of Web 2.0 conceptual and technical characteristics. Technical Web 2.0 characteristics refer, among other things, to rich application interfaces, syndication and micro content delivery. The conceptual features of the Web 2.0 paradigm emphasise on the collaboration and communication, providing great opportunities for citizen-centric service delivery, improved citizen participation and rich citizen connection between the citizen and the public sector. The adoption of the web 2.0 features in electronic Governance is discussed in [5] [6].

\section{B2G Interaction Modeling Based on a Web 2.0 Citizen-Centric Model}

A thorough governance model should take into account all types of governmental interaction ( $\mathrm{C} 2 \mathrm{G}, \mathrm{B} 2 \mathrm{G}, \mathrm{G} 2 \mathrm{G})$. The proposed model is focused on $\mathrm{B} 2 \mathrm{G}$ interaction, which is consecutively decomposed to multiple $\mathrm{C} 2 \mathrm{G}$ interactions. In fact, every interaction (B2G, G2G) is decomposed to $\mathrm{C} 2 \mathrm{G}$ interactions revealing a true citizen-centric model. The interaction model has been implemented as a social network with two kinds of relations: between citizens and between citizen and the government.

Government is an abstract term. The government sector consists of Public Agencies (PA) which are represented in the model through the Applications they provide. The design, the implementation, the development and the maintenance of the application is performed by the PA's IT sectors. PA Applications reside and executed in the corresponding PA IT infrastructure. 
Citizens are represented in the model through the Citizen's Profiles, which are considered as the fundamental components of the model. The importance of the citizen's profiles arises from the fact that every interaction with the governmental services has to pass through them. The Citizen Profile entity consists of three sections, namely Citizen, Business and Views as illustrated in figure 1.

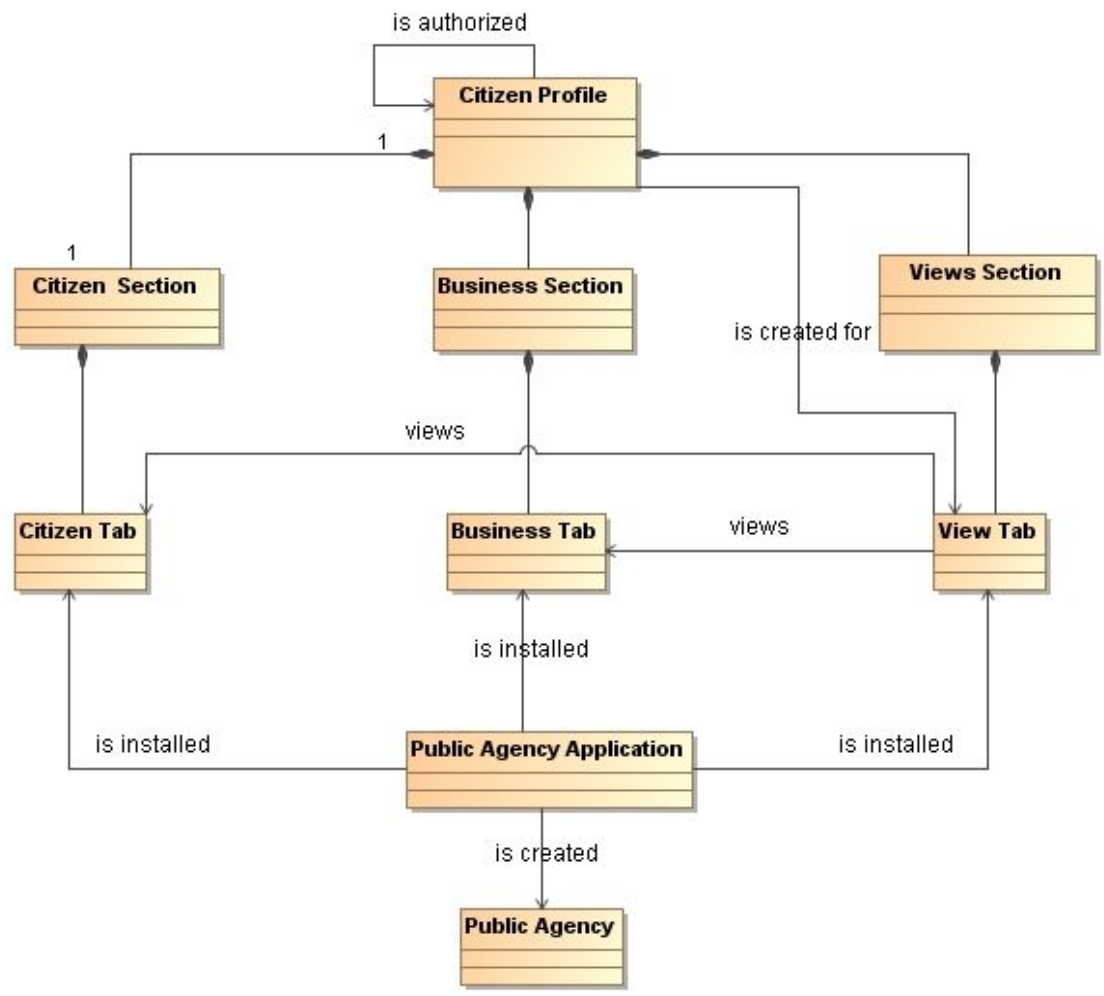

Fig. 1. A meta-model for Web 2.0 citizen-centric interaction

Figure 1 depicts the basic entities used to describe all types of interaction in a Web 2.0 citizen-centric fashion. Table 1, associated to the figure 1, further elaborates associations among the model's entities.

The Citizen Section of the Profile models Citizen to Government interaction. For consistency reasons the citizen section includes one and only Citizen Tab. The citizen tab can be considered as the personal data vault where the citizen can add information (by installing specific public agency applications) concerning his/her interaction with the government. For example, the citizen section could contain information about his/her studies, his tax status and so on. Cross organizational tasks can be accomplished by installing several applications from different public agencies and sharing the information that resides into the citizen tab among them. The installation of the applications into the citizen tab is depicted by the "is installed" association between the citizen tab and the public agency application. 
Table 1. Description of the association among Web 2.0 citizen-centric model entities

\begin{tabular}{|c|l|}
\hline Association & \multicolumn{1}{|c|}{ Description } \\
\hline is authorized & $\begin{array}{l}\text { A citizen profile can authorize another citizen profile. A formal } \\
\text { authorization scheme is adopted. }\end{array}$ \\
\hline is created for & $\begin{array}{l}\text { A view tab is created inside the views section of the intermediate } \\
\text { for the specific citizen profile (either citizen or business tag) when } \\
\text { the authorization takes place. }\end{array}$ \\
\hline is installed & $\begin{array}{l}\text { Applications can be installed in the citizen tab and in the business } \\
\text { tab. They can also be installed on behalf of the citizen tab or the } \\
\text { business tab and be administered in the view tab. }\end{array}$ \\
\hline views & $\begin{array}{l}\text { A view tab remotely administers some applications that consist a } \\
\text { view of the business tab or the citizen tab. }\end{array}$ \\
\hline is created & $\begin{array}{l}\text { Applications are created by the Public Agencies and are uploaded in } \\
\text { the platform registry. }\end{array}$ \\
\hline
\end{tabular}

The Business Section of the profile models Business to Government interaction. When a business is initiated, there must be a citizen declared as the legal intermediate of the business. This citizen is liable to governmental laws, acts and regulations as far as the business is concerned. Upon initialization of the business a Business Tab will be added inside the Business Section of the Citizen Profile. A citizen can administrate multiple businesses, so multiple business tabs can exist inside the business section. The citizen should be able to add applications on "his/her" business tab, to accomplished tasks on behalf of the business. This is the most simplified Business to Government interaction. In the real world, this interaction is more complex, as a business (or more accurately the citizen that is in charge of the business) often declares other citizens to accomplish tasks on behalf of the business. The interaction becomes even more complex when a business declares another business to represent it and accomplish governmental tasks. Thus, the notion of the Business or even Citizen Representative, as an intermediate, should be supported by the model.

The Views Section of the profile is introduced for that purpose. The Views section is decomposed to View Tabs that are instantiated when the citizen is asked to perform a task on behalf of another business or citizen, as an intermediate. An authorization procedure among citizen profiles is followed, the outcome of which is the creation of customized view tabs ("is created for" association of Table 1). The authorization scheme which is adopted requires a notification of the citizen profile and the explicit consent of the citizen to that request ("is authorized" association of Table 1). Consequently, the authorized citizen can install applications in the view tab that administers. Conceptually, the application is installed and executed in the business tab of the citizen that triggered the authorization. The authorized citizen remotely administers some applications that consists a view of the business tab ("views" association of Table 1). The output of the application is loaded on the business tab, which acts as a reference point for the coordination, the information extraction and the control of the applications, and is viewed or accessed by the view tab. The decomposition of business tab to views is also necessary to preserve sensitive business data and to enforce control over 
business processes. When cross organizational tasks are involved coordination of all the citizens that administer the business tab of the business should be supported.

Citizen to Citizen interactions to accomplish governmental tasks are also modeled. A citizen can authorize another citizen or business (more accurately a citizen that administers a business). The authorized citizen will act, on behalf on the citizen, by adding applications into the view tab of the citizen. The authorization process can involve more than one step. A citizen can authorize another citizen which authorizes another citizen to accomplish the same or part of the initial task and so on. The model is working, as the reference point for the coordination remains the business and the citizen tab.

To conclude, citizens may have multiple properties, as in the real world. They are mainly citizens with their attributes (Citizen Section), they can also administer a business that requires interaction with the Government (Business Section) and finally can accomplish a task on behalf of another citizen or business as a properly authorized intermediate (Views Section). Conceptually, a social network is created. Special effort was placed so as the model to be simple, accurate and consistent. Citizens provide the content to the platform, using the necessary applications. It is up to them to decide whether they will share their private content with other citizens or public agencies to accomplish complex or cross organizational tasks.

Simplicity can be considered as another key feature of the model. As, it is mentioned in the "Laws of Simplicity" book the "the simplest way to achieve simplicity is through thoughtful reduction" [7]. The model was intentionally designed without complex Government to Government integration schemas leaving the interaction workflow to the citizens

\section{MyCPP Platform}

To support the suggested Web 2.0 citizen-centric interaction model, a corresponding platform, named $\mathrm{MyCCP}$, is proposed. Its purpose is to provide an easy to use interface for citizens to built and manage their profiles, using Web 2.0 technology, while at the same time it provides for PA application registration and integration in a concise manner.

Applications are developed and deployed by public agencies, integrated in MyCCP platform and finally delivered to citizens, operating as MyCCP users. From the technological perspective, key technical features of the Web 2.0 paradigm such as rich application interfaces, syndication and micro content delivery should be integrated within MyCCP to enhance service delivery to citizens. From the public agencies point of view, applications are running on their IT infrastructure and invoked as web services based on the concepts of Web Oriented Architectures (WOA) [8]. Representational state transfer protocol (REST) [9] has been adopted as the architectural style of API development. Applications should be authenticated by the platform and registered in an application registry. Both citizens and PA IT personnel, uploading applications, should be properly authenticated using the necessary certificates to enhance privacy and trust. 


\section{1 Architecture}

MyCCP platform architecture is depicted in figure 2. It consists of three layers: integration, interaction and application layer. The integration layer provides the necessary low level functionality for the Public Agencies to develop and integrate applications within the platform. The interaction layer provides built-in services for the citizens to interact with the applications in a safe and easy to use environment. It also consists of the internal management modules that facilitate the profile, the applications, the relations and the trust mechanisms. The application layer is used, in conjunction with the integration and interaction layer to provide applications to the citizens.

\subsection{Integration Layer}

This layer enables applications to be easily integrated into the platform, in a seamless fashion. It also addresses issues related to Authentication, Data Integrity and Availability. While confidentiality deals with the unintentional disclosure of information outside the authorized parameters, data integrity assures the trustworthiness of the information and availability ensures that the information is made available to requesting authenticated clients. The integration layer is based on a platform-specific $A P I$ and a mark up language $(M y C C P M L)$. Specific client libraries provide a functional wrapper for the Platform's API REST interface. The main concern of the API is to facilitate a secure pull and push mechanism for the data process. The mark-up language ( $M y C C P M L)$ contains the required tags to implement citizen's profile. Thus, it contains presentation and semantic tags facilitating citizen-related data presentation and exchange between public agency applications. The special tags are used for the accurate acquisition of the profile information.

\subsection{Interaction Layer}

The interaction layer is in the core of the platform. It provides built-in functionalities for the citizens to interact with the platform and consequently PA applications. The interaction layer consists of two sub layers: The built- in citizen services sub-layer provides the necessary functionality for the citizen to interact with the platform. Based on the citizen requirements five services have been identified.

The log-in and authentication module provides citizen authentication. Only citizens can log-in in the platform. Alternative interaction models usually provide a username and a password to a business thus permitting a "business" to log-in and interact with the government. We argue that this concept is inappropriate. Businesses should be decomposed to the citizens that work for that business, so the citizen interaction with the government should be modelled. Upon log-in, citizen can access the citizen the business and views section of their profile. Certain policies can be adopted based on the technological background of the citizens including smart cards and certificates issued by a certification authority. HTTP/SSL mechanism provides the appropriate log-in security. 

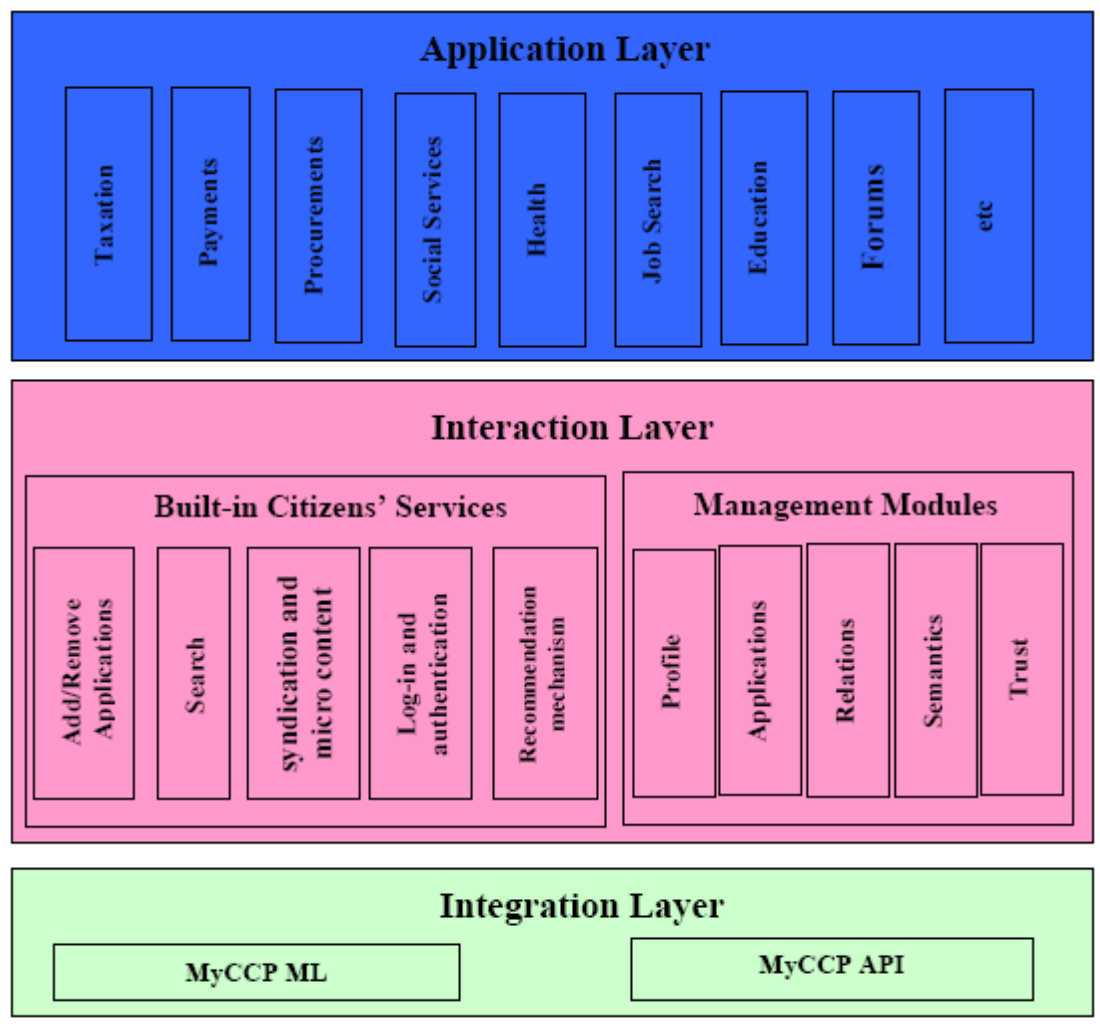

Fig. 2. MyCCP platform architecture

The add and remove applications module provides the necessary functionality for an application to be installed and uninstalled in the citizens profile in a seamless way. A citizen can add an application in the citizen and in the business section of his profile. Additionally, can remotely add and execute an application on behalf of another citizen or business. This sort of interaction takes place in the Views section. It is noteworthy to mention that, restrictions regarding the addition and the execution of the applications are handled solely by the applications. For instance, if an application is required to be added only by accountants, it is the application task to check the appropriate accountant registries and allow or not the addition of the application. The add and remove applications service is used complementary to the Recommendation mechanism service.

The Recommendation mechanism is part of the personalization features provided by the platform. The recommendation mechanism assists the citizen to arrange his/her profile, for example install the necessary applications to accomplish a task. Upon removal of an application, this module notifies the citizen for the possible implications on the execution of the depended applications. The recommendation mechanism is a complex service. It requires semantic information to identify the prerequisite applications. The decomposition of the business to the intermediate citizens also 
poses new requirements. For instance, an application executed by an intermediate citizen may require a prerequisite application that should be executed by another intermediate citizen. The recommendation mechanism, in conjunction with the relation module should handle the interaction between them.

A search module enables citizens to search for the appropriate applications included in application registry. Semantic information is stored for each application, providing the documentation for the citizens to find the right applications and properly combine them so as to complete a complex cross organizational task.

Syndication and micro content delivery module alerts citizens for particular issues that may concern them. It also notifies the intermediate citizens for the pending tasks. Pending tasks can concern the addition and execution of certain applications.

Along with the Built in citizens' services sub layer, the Management Modules sub layer exists. Management modules implement and administer the fundamental functionality of the platform.

The profile management mechanism updates citizens' profiles based on the applications they have installed. As already stated, the citizen profile consists of three sections, namely Citizen, Business and Views. Consequently, the sections are decomposed to tabs. Applications are added inside the tabs. The profile management mechanism is also responsible to replicate information contained in view sections to the citizen and business section. The administration of the tabs and the sections is handled by the profile management mechanism.

The relation management administers any kind of relations in the model. The model requires interaction among the intermediate citizens. The relation mechanism handles the authorization process, that is the process that affiliates a citizen to a business. When a complex cross organizational task is required to be accomplished, relation management searches for the intermediate citizens that are related with the business. Consequently, utilizes the Syndication and micro content delivery module and notifies the citizen about the pending jobs.

Trust mechanism facilitates rating of public services and provides feedback to other citizens and the public sector.

Application management mechanism is responsible for ensuring the registration, the authentication and the availability of the applications. An application registry will be utilized for that purpose. Applications are developed by the public agencies to provide specific services to the platform. The distributed nature of the model requires that applications are implemented unaware of the existence of the other applications that may have been implemented. Application registry requires some necessary information for the application to be uploaded such as the public agency, the category of the application (e.g finance), the date that was added to the registry, a description of the application, some tags to identify the use, the preconditions (input) that are required for the application to execute and finally the output information that the application will add to the citizen profile. Some additional information may also be required by the registry, e.g. for security reasons.

The output information that is posted from an application should be discovered and used, upon citizens' explicit consent, as prerequisite information for another application. Thus, the output information should be semantically tagged in a proper and coherent way. 
The semantics module is responsible for the semantic tagging, which is not performed in an automated manner. Semantic annotation in a decentralized Web 2.0 environment is hampered by the vocabulary problem and the language gap [10]: an application requires some preconditions as input information, but is unable to use the right terms to search for it and there is a lack of common semantic descriptions of available output information. To this end, a collaborative recommendation tagging system is proposed to implement semantics module. When registering an application, the corresponding Public Agency is forced to provide tags upon output usage and functionality forming tag-clouds per Public Agency or Category. Tag clouds refer to aggregated tag information in which a taxonomy or "tagsonomy" emerges through repeated collective usage of the same tags.

When the output information of the application is about to be semantically described, the collaborative recommendation system will recommend the most frequently used tags that have been added by Pubic Agencies included in the same category. For example, when the majority of the Universities have tagged the output of their "Degree Certificate" application as "degree rate", the collaborative recommendation system will recommend that tag instead of the "grade rate" used by a specific University, leading to more consistent semantic tagging. The tag cloud mechanism will also facilitate Public Agencies to select the proper preconditions for their applications from those already included in the registry, by minimize tag range. For example, the collaborative recommendation system will notify the public agencies involved that the most frequently used tags in the category "universities" are the "degree rate" and the "grade rate". Consequently, it is up to the public agencies to decide whether they will require as precondition the "degree rate", the "grade rate" or both, it they have similar meaning. In the conceptual level, Public Agencies formulate a social sub-network where collaborative tagging provides a collective intelligence to the platform.

\subsection{Application Layer}

The Application Layer provides a set of interface specifications to support various types of applications in every administrative level (Local, Federal, European). Public Agencies' databases and business logic will remain intact. However, a front end interface will have to be implemented using the platform's API and platform's markup language. Then, the application will have to be tagged with semantic information and be uploaded in the registry. Applications can be considered as the main execution component of the platform. They act as gateways between the citizens and the public agencies, providing a fundamental engagement mechanism. Applications can provide governmental services from the whole public sector spectrum including taxation and payment, procurements, social services, health, job and education.

\section{Case Study}

To demonstrate the potential of the proposed interaction model a cross-organizational business-to-government example is discussed in the following. Let's assume a midscale software company, called Synapses and a Public Agency making a call for proposals to purchase software. According to EU legislation, any proposal should be 
accompanied with certifications proving that the company's tax and insurance status is clear. The founder and the CEO of the company, named Jason, has a business tab with the name of the company added inside the business section of his profile. So, Jason's Citizen Profile currently consists of the Citizen Section that includes his Citizen Tab and the Business Section that includes Synapses Business Tab. Jason wishes to authorize Alice as the accountant and Bob as the lawyer, while both work as freelancers representing potential client when interaction with the government. Jason also wishes to authorize Charlie, an employer, as a intermediate to place offers for public calls. When Alice, Bob and Charlie accept their roles, a view tab, named Synapses, is added to their citizen profile inside the Views Section.

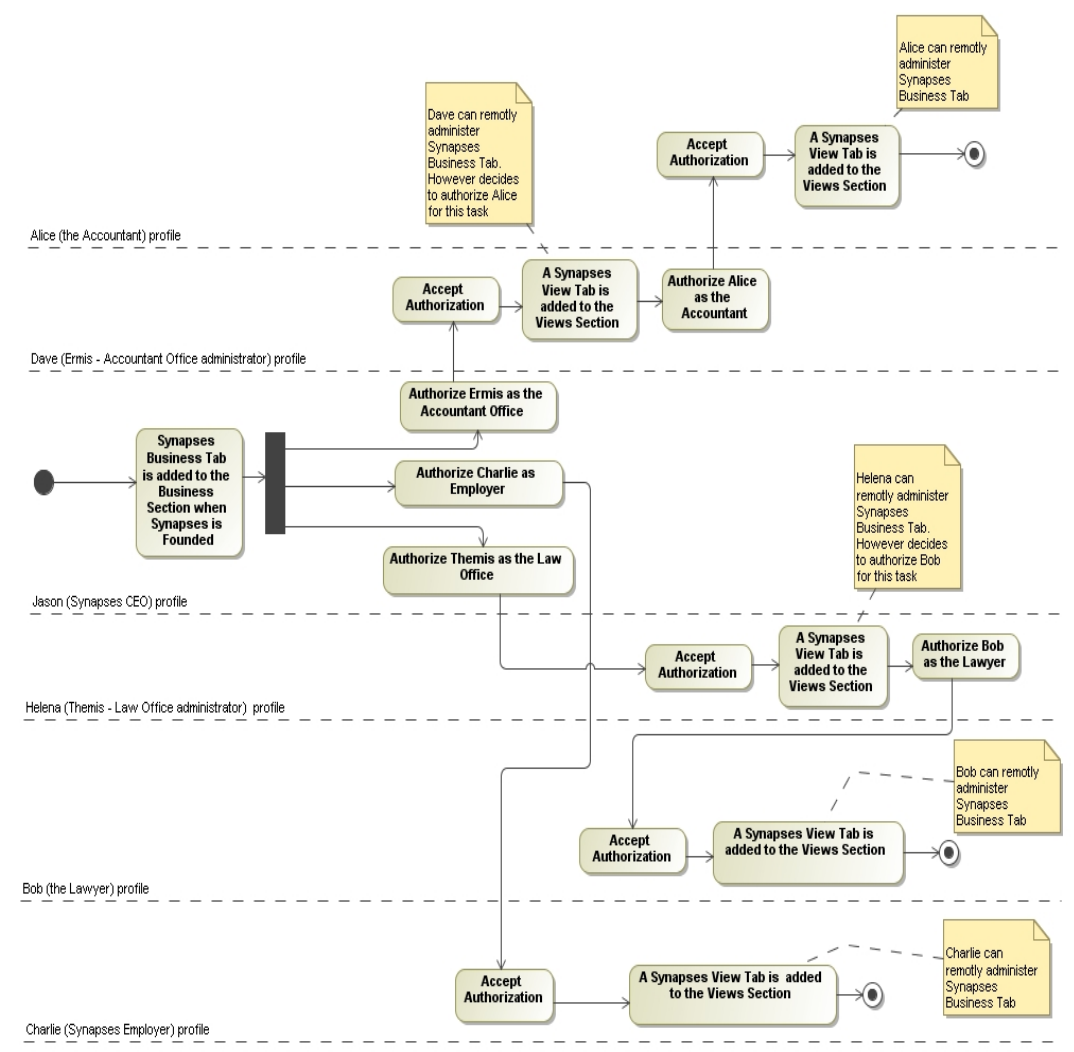

Fig. 3. Authentication Procedure

A more complex interaction takes place when the Jason authorizes, instead of citizens, another business. Let's suppose that Jason authorizes an accountant office called Ermis to take care of the accountant and financial tasks of "his" business. He also authorizes Themis as the law office to accomplish tasks for Synapses. Ermis, is administered by a citizen called Dave and Themis is administered by a citizen called Helena. They both accept the authorizations and a view tab, named Synapses, is added to their citizen's profile. Suppose that Alice the accountant is working for 
Ermis and Dave authorizes her to elaborate on Synapses' tax issues. Alice will accept Dave's authorization on Synapses, which means that Alice is able to administer Synapses Business Tab. A Synapses View Tab will be added in her profile. In the exact same way, Bob, who is working for Themis, is authorized as the lawyer for Synapses. The authorization procedure is depicted in figure 3 .

The public Agency has created an application that requires a clearance certification from both the tax office (Ministry of Economy and Finance) and the National Insurance Company (Ministry of Labor) and consequently processes the proposals. Charlie installs that application and the recommendation mechanism informs him about the prerequisites. Charlie does not know who is responsible to install and run the necessary applications, so the platform searches Synapses business tab and alerts every citizen that views some part of it for the pending tasks. This is actually performed by the relation module of MyCCP.

Practically, relation module will check the Synapses business tab on Jason's profile to find the corresponding authorized citizens, which are Dave and Helena (as the accountant and law office administrators) and consequently Alice and Bob. The information that was posted in Synapses business tab located on Jason's citizen profile will be replicated to Synapses' view tab located on the Dave's citizen profile and

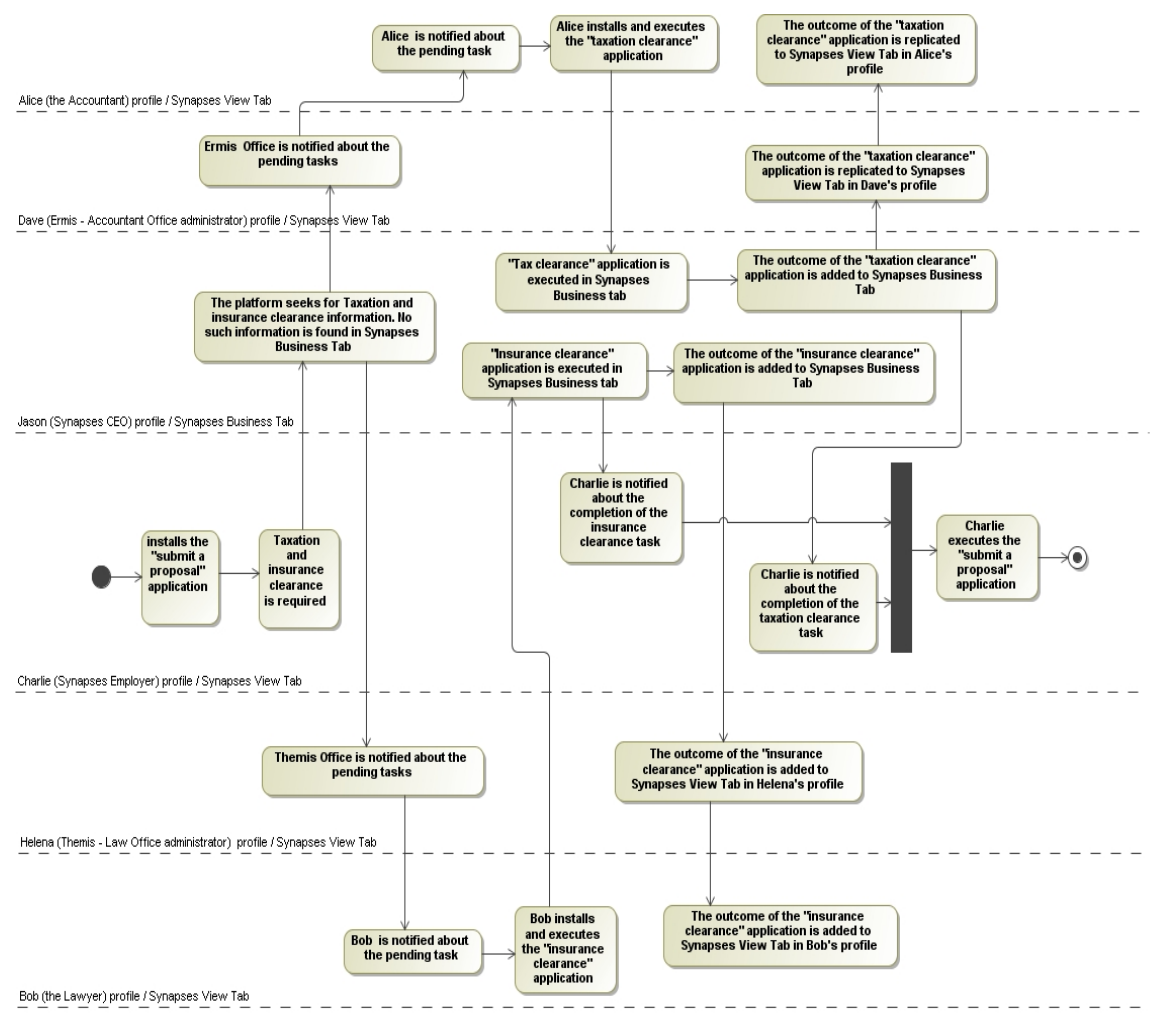

Fig. 4. The "Submit proposal for purchasing software" Procedure 
consequently to Synapses view tab located in the Alice's citizen profile. Alice will be notified about the pending task and will add in Synapses business tab in the views section of her profile the appropriate application from the Ministry of Economy and Finance that will verify that Jason's company has paid the necessary taxes.

In a similar fashion, Bob the Lawyer will be notified by Themis, which will be notified by Synapses. The information will be posted to Synapses business tab located on Jason profile, will be replicated to Synapses view tab located on Helena's profile and finally be replicated to Synapses view tab located on Bob's profile.

Both applications will be actually executed in the Synapses business tab of Jason's profile and will be ultimately replicated in Alice's and Bob's corresponding tabs in the views section of their profile. Note, that Bob is unaware of Alice's application and so does Alice for Bob's. Only Jason, as the business tab owner, has a full report of the information that was posted in the view tabs. Charlie is notified that the pending tasks are completed and accomplishes the proposal submission. Synapses business tab in the Business Section of Jason's profile is the reference point for every interaction. Corresponding profile interaction is illustrated in figure 4.

\section{Conclusions}

The proposed Web 2.0 citizen-centric model has been designed to provide novel interaction between Business and Government. This novel approach relies on the citizens' will to provide and share certain information with public agencies and his/her active participation in workflow execution of complex cross-organisational tasks, combining the functionality of discrete public agencies applications. Business information is integrated to the citizen profile, in autonomous business tabs, contributing to the establishment of a sophisticated and homogenous interaction model. The notion of intermediates acting as representatives of a citizen or a business tab is also integrated in the model. This functionality was implemented by introducing the View Tabs, which enable citizens to control parts of another citizen profile. Ultimately, all kinds of interactions (B2G, G2G) are expressed as $\mathrm{C} 2 \mathrm{G}$ interactions revealing the citizen-centric nature of the proposed interaction model. The model is accompanied by a corresponding Web 2.0 platform, called MyCCP.

Future work involves the implementation of a prototype. The platform API libraries should be implemented and the semantic and presentation tags of the MyCCP mark up language should be defined. In addition, security issues should be thoroughly examined. Finally, semantics management should be further explored, in terms of the collaborative recommendation system. Finally, some issues concerning the cold start problem and the adoption or not of existing e-gov ontologies have to be resolved.

\section{References}

1. Christensen, T., Lægreid, P.: The Whole-of-Government Approach to Public Sector Reform. Public Administration Review 67(6), 1059-1066 (2007)

2. Momotko, M., Izdebski, W., Tambouris, E.s., Tarabanis, K., Vintar, M.: An Architecture of Active Life Event Portals: Generic Workflow Approach. In: Wimmer, M., Scholl, J., Grpnlund, E. (eds.) EGOV 2007. LNCS, vol. 4656, pp. 104-115. Springer, Heidelberg (2007) 
3. Sahraoui, S., Ghoneim, A., Irani, Z., Ozkan, S.: T-Government for benefit realisation: A research agenda. In: Evaluating information Systems. Public and Private Sector, pp. 359-373. Butterworth-Heinemann/Elsevier (2008)

4. Oreilly, T.: What is Web 2.0: Design Patterns and Business Models for the Next Generation of Software, http://oreilly.com/web2/archive/what-is-web-20.html

5. Maio, A.: Government and Web 2.0: The Emerging Midoffice. Gartner (2007)

6. Maio, A.: The E-Government Hype Cycle Meets Web 2.0. Gartner (2007)

7. Maeda, J.: The Laws of Simplicity (Simplicity: Design, Technology, Business, Life), pp. 73-81. MIT Press, Cambridge (2006)

8. Sholler, D.: Reference Architecture for Web-Oriented Architecture. Gartner (2008)

9. Fielding, R., Taylor, R.: Principled Design of the Modern Web Architecture. ACM Transactions on Internet Technology (TOIT), 115-150 (2002)

10. Fernandez, A., Hayes, C., Loutas, N., Peristeras, V., Polleres, A., Tarabanis, K.: Closing the Service Discovery Gap by Collaborative Tagging and Clustering Techniques. In: ISCW 2007, Workshop on Service Discovery and Resource Retrieval in the Semantic Web (2008) 